\title{
L'effondrement de 1873 à la mine de Varangéville
}

Laboratoire de Mécanique

\section{P. BÉREST}

École Polytechnique

91128 Palaiseau Cedex berest@lms.polytechnique.fr

\section{B. BROUARD}

Brouard Consulting 101, rue du Temple 75003 Paris

B. FEUGA

Geoderis, BP 25198

57075 Metz Cedex 3

\section{KARIMI-JAFARI}

Laboratoire de Mécanique des Solides actuellement Geostock 7 , rue Eugèneet-Armand-Peugeot 92500 Rueil-Malmaison

\begin{abstract}
'Q On relate l'effondrement d'un quartier de la mine de sel de Varangéville survenu en 1873. Les descriptions de l'époque montrent que la partie centrale du recouvrement de la mine est descendue en bloc, entraînant une couronne intensément déformée. Les piliers ont poinçonné précocement le mur imbibé de saumure, mais le toit les a retenus tant que l'extension de la mine, et donc sa raideur à la flexion restaient modérées. Le calcul numérique montre que le caractère brutal de l'effondrement peut être relié au développement d'une zone dilatante, traversant toute la couche de sel, et engendrée par le report sur le contour de la mine d'une part du poids des terrains surincombants.
\end{abstract}

Mots-clés : mine de sel, chambres et piliers, dilatance, effondrement.

\section{The 1873 collapse at the Varangéville Salt Mine}

The 1873 collapse of a panel of the Varangéville Salt Mine is described. The central part of the overburden dropped down as a rigid block; between this cylinder and the intact rock mass, a rock crown was severely deformed. The marly floor of the mine, which had been weathered by water, were punched by the mine pillars at an early stage; however mine roof prevented full punching as long as roof extension, thus roof bending stiffness, remained small. Numerical computations prove that the catastrophic character of the collapse can be explained by the development of a dilatant zone, crossing through the salt layers, which had formed upon the mine edge, as a part of the overburden weight was transferred to the abutment.

Key words: salt mine, rooms and pillars, dilation, collapse. 


\section{Introduction}

Dans l'après-midi du 31 octobre 1873, le quartier Saint-Maximilien de la mine de sel de Varangéville s'ef fondra brutalement. La secousse fut ressentie à Nancy, distante d'une dizaine de kilomètres. L'effondrement vint par surprise, car les piliers de la mine étaient jugés très sains. Toutefois des grondements s'étaient fait entendre le matin dans la mine et une fissure était apparue dans un bâtiment à la surface du sol, de sorte que le quartier avait été évacué dans la matinée. De plus, c'était jour de paie, de sorte que peu de mineurs se trouvaient au fond, et le nombre de victimes fut limité.

\section{2}

\section{Observations faites après l'effondrement}

\section{9.}

\section{Surface du sol}

A la surface du sol, une cuvette de subsidence s'était formée (Fig. 1). Plusieurs rangées concentriques de fissures verticales ouvertes, peu profondes, délimitaient une (c ellipse extérieure ), d'un rayon de $160 \mathrm{~m}$ environ, centrée autour du puits $n^{\circ} 1$ et inscrite à l'intérieur du périmètre extérieur des travaux miniers. Ces fissures témoignaient des effets de contraintes horizontales de traction intenses. Des bourrelets, signes de contraintes horizontales de compression, s'étaient formés sur le pourtour d'une « ellipse intérieure » de rayon moitié. L'intérieur de cette ellipse était descendu uniformément de 3,3 mètres. Entre les ellipses intérieure et extérieure, la surface du sol avait pris une inclinaison régulière en direction du centre de la zone affaissée ; la pente était de l'ordre de $3,3 / 80=4 / 100$. Le puits $n^{\circ} 1$, d'une profondeur de $160 \mathrm{~m}$, était intact, mais son fond était rempli sur les derniers 18 mètres par des débris divers, dont des marnes remontées du mur de la mine.

\section{9.}

\section{Désordres au fond}

La mine était ouverte, entre 150,5 et $156 \mathrm{~m}$ de profondeur, à la base de la $11^{\mathrm{e}}$ couche de sel. La formation salifère appartient au Keuper inférieur de Lorraine. Les géologues l'ont divisée (Fig. 3), depuis le toit du sel qui est à 80 mètres de profondeur jusqu'au mur de la mine qui est profond de 160 mètres, en 11 couches de sel séparées par 10 niveaux marneux dont l'épaisseur varie de 0,5 à $3 \mathrm{~m}$. La $11^{\mathrm{e}}$ couche a une épaisseur d'une vingtaine de mètres et sa base contient le sel le plus pur. Sous la $11^{\mathrm{e}}$ couche on trouve une couche marneuse, de $25 \mathrm{~m}$ d'épaisseur ou plus, décrite en plus grand détail par la suite. La mine était exploitée par la méthode des chambres et piliers. Les chambres avaient $5,5 \mathrm{~m}$ de haut. Des galeries parallèles et perpendiculaires, de $8 \mathrm{~m}$ de large dans la direction SO-NE et de $9 \mathrm{~m}$ de large dans la direction perpendiculaire, laissaient des piliers carrés de $6 \mathrm{~m}$ de côté, portant le taux de

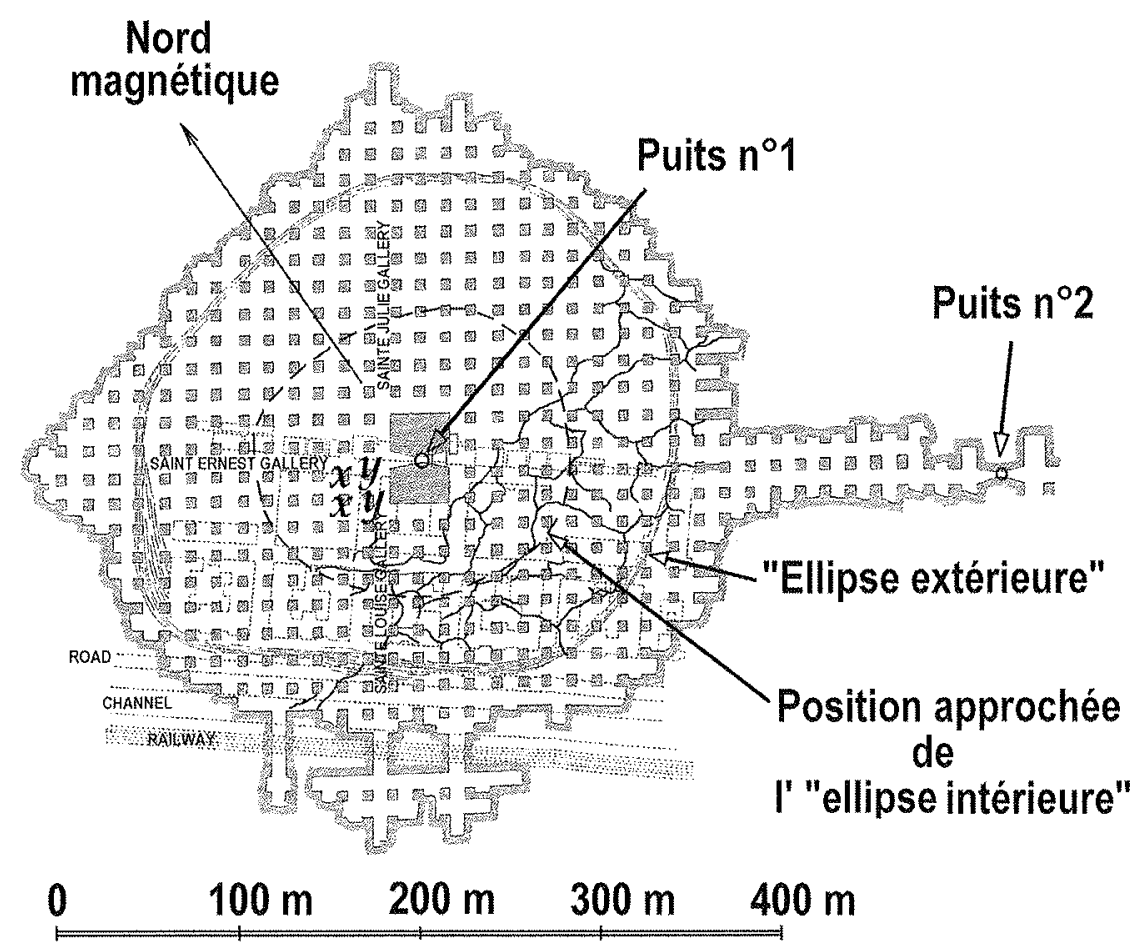
la surface lors de l'effondrement, dessiné le $1^{\text {er }}$ novembre 1873. On a reporté le réseau polygonal des fentes verticales remplies de marnes, les bâtiments de surface sont en pointillés (d'après Braconnier, 1873a).

Subsidence trough above the Saint-Maximilien panel as observed on November 1st, 1873. Cracks along the outer ellipse, building locations at ground level, pillars and the irregular pattern of vertical mud-cracks at panel level also were reported (after Braconnier, 1873a). 
défruitement à $\tau=1-(6 \times 6) /(14 \times 15)=82 \%$, un taux considéré à l'époque comme raisonnable. Toutefois un pilier rectangulaire de $29 \mathrm{~m} \times 40 \mathrm{~m}$ avait été ménagé au centre du quartier pour protéger le puits $n^{\circ} 1$ (Fig. 1).

Les $1^{\text {er }}$ et 2 novembre des mineurs et Marie-Alfred Braconnier, ingénieur des mines qui a laissé des descriptions détaillées de l'accident, descendirent par le puits $n^{\circ} 2$ pour visiter les travaux effondrés.

( Sous l'ellipse intérieure à mi-distance entre le puits $n^{\circ} 1$ et le contour général de l'exploitation souterraine, les piliers de sel se sont enfoncés dans les marnes, celles-ci se sont élevées dans les galeries jusqu'à toucher le toit, lequel, en s'affaissant, paraît avoir conservé presque toute sa solidité primitive et n'offre que des fissures [...]. Le sol des galeries intermédiaires entre les deux ellipses n'est jonché d'aucun débris. Il présente une pente régulière ascendante, le toit offrant au contraire une pente régulièrement descendante. Les piliers se sont d'autant plus inclinés en pénétrant dans les marnes qu'ils étaient plus éloignés de l'ellipse intérieure ) (Braconnier, 1873b, p. 1).

Avant l'accident, selon Braconnier, les piliers de sel de la mine donnaient une impression de grande solidité. Sous la couronne déformée, ils ont, d'autant plus profondément qu'on s'approche du pilier central, poinçonné le mur, qui s'est incliné vers le haut dans la direction du puits central (Fig. 2). Ils sont globalement intacts, quoique leurs angles aient éclaté et que des fissures initialement fermées se soient ouvertes, certaines avant l'accident, en suivant des fentes verticales remplies de marnes, de sel et d'anhydrite, contemporaines du dépôt, et qui constituent des surfaces de faiblesse mécanique. En revanche le toit des galeries périphériques (à gauche sur les figures 2 et 4) était très dégradé :

« Le long du contour de l'exploitation, les parements latéraux des galeries étaient restés verticaux; mais des blocs énormes en forme de dalles étaient tombés du toit, de sorte cu'il existait, au faîte de ces galeries, des cloches ayant jusqu'à 3 mètres de haut. [...] ; les parois de ces cloches présentaient des fissures béantes... On peut conclure de là que, sur tout le pourtour de la mine, le terrain a été soumis à une compression dans le sens horizontal... ) (Anonyme, 1873, p. 625).

En 1855 , la $4^{\mathrm{e}}$ couche de sel, à une profondeur de $87 \mathrm{~m}$, avait aussi été exploitée, à partir du puits $\mathrm{n}^{\circ} 1$ et au-dessus de la future exploitation en $11^{\mathrm{e}}$ couche. L'effondrement de 1873 n'a engendré aucun dommage dans ce quartier, si ce n'est quelques fissures, à une distance de $80 \mathrm{~m}$ de l'axe du puits, desquelles s'écoulait un petit débit de saumure. Un bure et une galerie de section $3 \mathrm{~m}$ × 3 m furent creusés plusieurs années après l'effondrement pour relier les exploitations de $4^{\mathrm{e}}$ et $11^{\mathrm{e}}$ couches. Cette galerie permet d'observer le massif de sel quelques mètres au-dessus du quartier effondré. Le massif est dans l'ensemble intact mais des petites fractures, qui permettent à l'air de circuler entre la galerie et les travaux effondrés, peuvent être observées sur une longueur d'une dizaine de mètres, exactement au-dessus des galeries du contour de l'exploitation.

\section{3 \\ Cinématique de l'effondrement}

Les trois rapports qu'a laissés Braconnier (1873a, b, c) suggèrent donc fortement le schéma d'effondrement suivant : autour du puits central $n^{\circ} 1$, sous l'ellipse intérieure, un cylindre de hauteur $160 \mathrm{~m}$ et de rayon voisin de $80 \mathrm{~m}$ est descendu en bloc, de 3,3 mètres, sans pratiquement se déformer, en poinçonnant le mur de la mine (Fig. 3). Ce cylindre a entraîné en tombant un anneau de terrains à sa périphérie, entre 80 et $160 \mathrm{~m}$ de distance à l'axe du puits, qui est d'autant plus descendu qu'on s'approche du cylindre central.

Ce mécanisme est confirmé par l'ensemble des données déjà présentées - les chiffres renvoient à la figure 3 - (1) à l'intérieur de l'ellipse centrale, le sol est resté plat; (2) le puits est resté pratiquement intact; (3) le petit quartier exploité dans la $4^{\mathrm{e}}$ couche en 1859 , à $70 \mathrm{~m}$ au-dessus de l'exploitation de $11^{\mathrm{e}}$ couche, est resté intact, à l'exception de quelques fissures à $80 \mathrm{~m}$

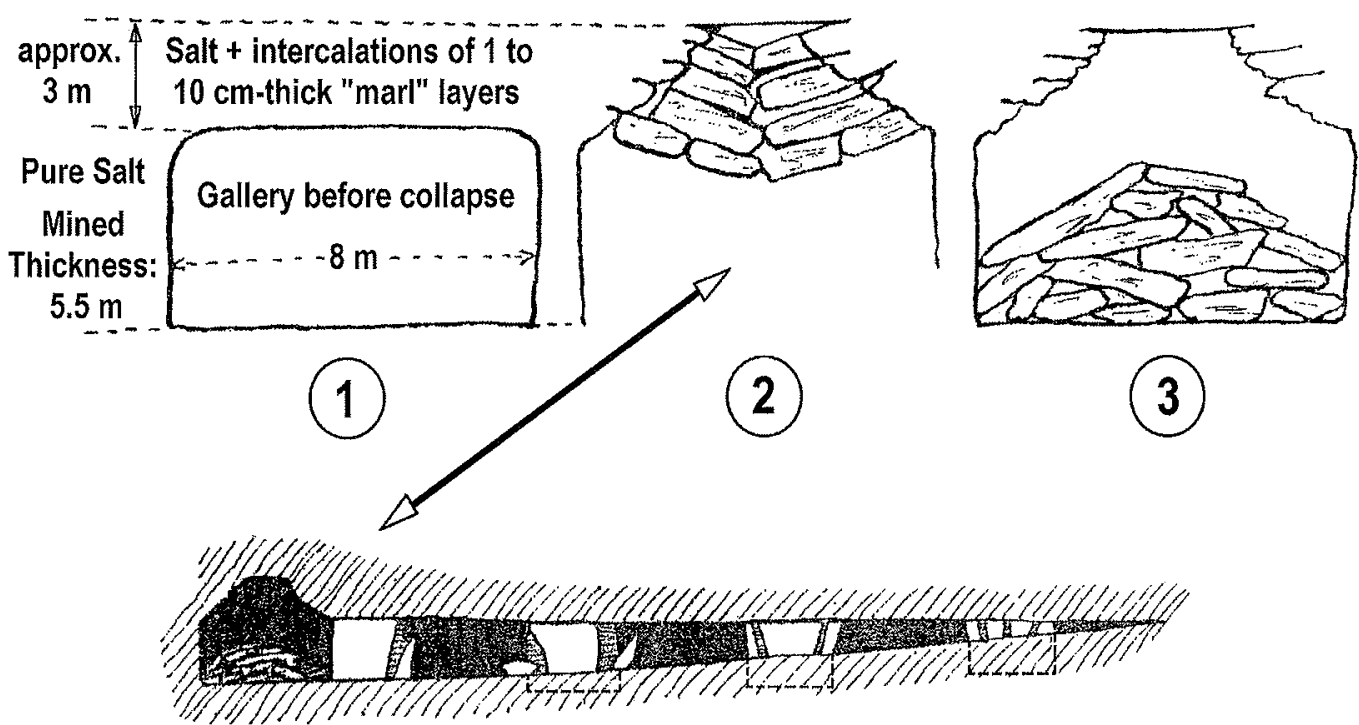

IIG 2 Coupe verticale des trois galeries les plus extérieures et phases successives de

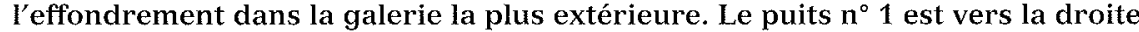
(d'après Braconnier, 1873c).

Vertical cross-section of the three outmost galleries and sequence of events in the outmost gallery (after Braconnier, 1873c). 


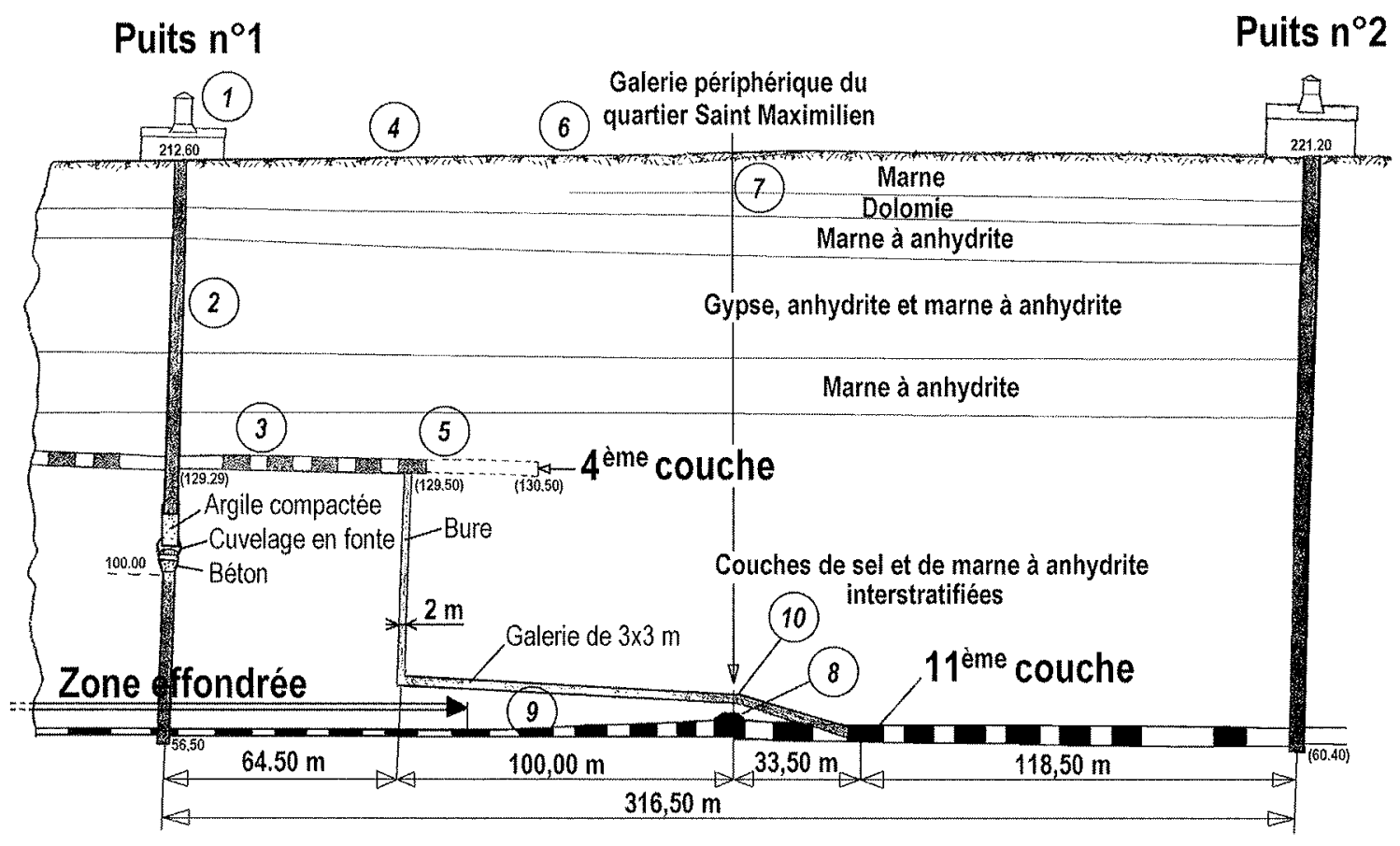

16. 3 Coupe verticale des travaux montrant les quartiers de $11^{\mathrm{e}}$ et $4^{\mathrm{e}}$ couches et la galerie $3 \mathrm{~m} x$ $3 \mathrm{~m}$ creusée après l'accident (d'après Braconnier, 1873c).

Rock mass cross-section showing the 11th and 4th layers panels and the $3 \mathrm{~m} \times 3 \mathrm{~m}$ gallery excavated after the accident (after Braconnier, 1873c).

de l'axe du puits. En revanche la couronne comprise entre ce cylindre central et le cylindre qui s'appuie sur le contour extérieur du quartier, entre $80 \mathrm{~m}$ et $160 \mathrm{~m}$ de distance à l'axe du puits, s'est déformée. Cette déformation doit être complexe dans le détail, les terrains étant stratifiés. Néanmoins on peut grossièrement la décrire, dans un plan méridien vertical, comme une flexion. En effet (4) des bourrelets indices d'une compression horizontale se sont formés en surface sur le pourtour de l'ellipse intérieure ; (5) de petites fissures, mentionnées en (3), sont apparues à la périphérie de l'exploitation de $4^{e}$ couche; (6) le sol a pris une pente régulière entre les deux ellipses ; (7) des fractures indices d'une traction horizontale se sont formées sur le pourtour de l'ellipse extérieure; (8) à $160 \mathrm{~m}$ sous ce pourtour, une montée de voûte sur 3 mètres de haut et des déformations horizontales intenses ont été observées (Fig. 4). Le reste du toit du quartier s'est incliné en direction du puits $n^{\circ} 1$, avec la même pente que celle de la surface du sol, de l'ordre de $4 \%$, en restant intact hormis des petites fissures (9). Dans la petite galerie de section $3 \mathrm{~m} \times 3 \mathrm{~m}$, creusée postérieurement à l'accident au-dessus du quartier effondré, le sel est intact sauf dans une zone d'une longueur de 10 mètres à l'aplomb des galeries du pourtour extérieur (10).

\section{4}

\section{Signes précurseurs}

\section{4}

\section{Désordres au fond}

On a dit que l'exploitalion avail élé arrêtée quelques heures avant l'effondrement, après qu'une fissure fut apparue dans un bâtiment et que des grondements se furent fait entendre au fond. En fait il y avait eu aupara-

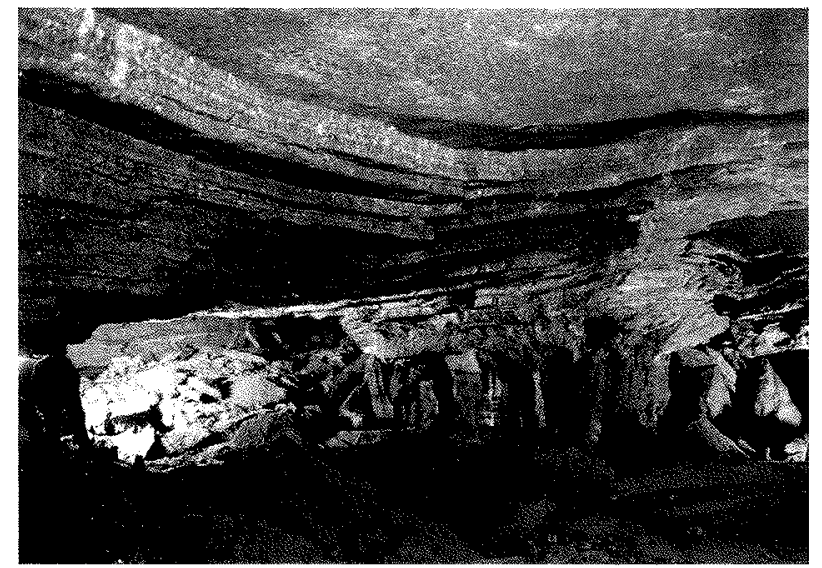

ro. 4 Toit et piliers dans les galeries les plus extérieures du quartier effondré (source : archives CSME).

Roof and pillar close to the external abutment in the collapsed panel (source : archives CSME).

vant quelques signes précurseurs ; ils sont particulièrement utiles pour les calculs numériques car ils permettent de se faire une idée des déplacements observés avant l'effondrement :

« Depuis 15 jours, à la surface, les mouvements d'affaissement, de contraction et de refoulement, insensibles sur la terre, se trahissaient par la rupture [...] de tuyaux de conduite en fonte. [...] Depuis cinq à six mois, le sol des galeries montait de manière sensible. Dans la galerie Sainte-Julie, nous constations le 20 octobre dernier que le sol avait monté de $0,80 \mathrm{~m}^{1}$. Un grand nom-

(1) « Il apparaît que le rétrécissement des galeries dans le sens de la hauteur s'était élevé jusqu'à 1,20 m. [1!] tenait non seulement à ce que le sol des galeries s'exhaussait mais aussi à ce que les piliers euxmêmes s'enfonçaient dans le sol n) (Braconnier, 1873c, p. 2). 
bre de piliers carrés présentaient sur toute leur hauteur des fentes atteignant jusqu'à $5 \mathrm{~cm}$. [...] La chambre de la machine à colonne d'eau [creusée dans le pilier central] n'était plus tenable. A la date précitée, elle s'élevait avec une vitesse de $6 \mathrm{~mm}$ par jour $»$.

De plus : « Dès l'année 1868, on avait remarqué que les piliers $x$ et y s'étaient fissurés et que la marne formant le sol des galeries se gonflait et se désagrégeait sous l'action de l'air et de l'eau ) (Anonyme, 1873, p. 620). Les quatre piliers $x$ et y sont situés près du croisement des galeries Saint-Ernest et Sainte-Julie, proche du pilier central (voir figure 1).

La rupture des tuyaux en fonte indique une subsidence au moins décimétrique. La réduction de hauteur de $80 \mathrm{~cm}$ de la galerie Sainte-Julie, et dans d'autres galeries proches du pilier central (Anonyme, 1873) est très suggestive. Le taux de défruitement est $80 \%$; en moyenne, les piliers doivent s'enfoncer de $64 \mathrm{~cm}$ pour faire monter le mur des galeries de $16 \mathrm{~cm}$ et produire finalement une réduction de hauteur des galeries de $80 \mathrm{~cm}$, sauf au voisinage du pilier central dont l'enfoncement est susceptible de fournir de grands volumes de marne aux galeries limitrophes. On ne peut imaginen qu'une zone de petits piliers s'enfonce de $64 \mathrm{~cm}$ sans que les zones voisines ne fassent de même (le toit n'y résisterait pas) ; c'est donc que le pilier central avait commencé à poinçonner le mur avant l'effondrement. Autrement dit, ce ne sont pas les petits piliers qui s'enfoncent autour de la galerie Sainte-Julie, mais le mur qui remonte.

\section{Le mur marneux}

Le comportement des marnes du mur avait attiré l'attention depuis longtemps:

« Cette couche de marne a probablement une épaisseur de plus de $20 \mathrm{~m}$. A la partie supérieure [...] elle s'est présentée d'une pureté extrême, nécessitant l'emploi de la poudre. Elle se compose de marne gris bleuâtre, très compacte, alternant avec de petites couches irrégulières de sel gemme rougeâtre, sillonnées obliquement par des filons très nombreux de sel gemme rouge fibreux, contenant des rognons irrégulièrement disséminés d'une substance très dure qu'on a toujours décrite comme de la marne solidifiée par du sel gemme et surtout par de l'anhydrite ») (Braconnier, 1873c, p. 1).

Sous cette croûte de marnes anhydritiques on trouve des marnes moins résistantes dites (c brun-chocolat ) dont l'épaisseur est de quelques mètres. Les deux types de marnes se dégradent quand elles sont en contact avec l'eau. Or l'eau était largement utilisée dans le quartier, notamment pour prédécouper le front de taille avant les tirs. Ainsi de la saumure non complètement saturée (sa concentration était estimée à $20^{\circ}$ Baumé, soit environ $250 \mathrm{~g} / \mathrm{l})$ s'infiltrait dans le mur :

« [...] au bout de 17 ans d'exploitation continue la quantité d'eau infiltrée dans la couche de marne a pu être finalement très considérable. Cette eau [...] a dû dissoudre partiellement les lits, filons et rognons de sel contenu dans les marnes. Elle a dû hydrater l'anhydrite qui en se transformant ainsi en gypse, développe un travail brisant extrêmement énergique. Elle a dû débiter enfin la marne même par l'action de l'oxygène qu'elle tenait en dissolution et qui a dû agir sur les sulfures et matières organiques que contient cette marne pure. Ces trois actions concouraient au même résultat qui a été l'amollissement général de la partie supérieure de la couche de marne et sa transformation en une masse spongieuse ») (Braconnier, 1873c, p. 1).

\section{5}

\section{Accidents de mines de sel}

\section{6}

\section{Ennoyage}

La majorité des accidents de mines de sel ont été provoqués par un ennoyage de la mine, plutôt que par une instabilité mécanique, même si un ennoyage a souvent pour conséquence un effondrement. Les mines de sel sont très vulnérables aux venues d'eau : une entrée d'eau initialement minuscule peut dégénérer en invasion incontrôlable. Bien entendu ces accidents ont aussi une composante mécanique, à leur origine ou pendant leur déroulement; mais c'est l'entrée de saumure claire dans la mine qui est l'élément déterminant. Quelques cas récents sont décrits par Bérest et al. (2004). Dans le cas de Saint-Maximilien, il est remarquable que l'effondrement ne se soit accompagné d'aucune venue d'eau dans le quartier, une circonstance qui reste vraie 135 ans plus tard, et établit qu'aucune fracture traversant le massif de sel n'a été créée.

\section{0}

\section{Rupture des piliers}

L'instabilité mécanique d'une mine de sel peut être provoquée par la rupture des piliers ; mais en général le sel est trop ductile pour que l'effondrement soit brutal. Minkley et Menzel (1996) ont montré que, dans la région de la Werra, en Thuringe (Allemagne), la destruction en 1989 de milliers de piliers en quelques secondes dans la mine de Voelkershausen, déclenchée par un tir, s'expliquait par la fragilité extrême du minerai exploité (carnallitite) et le taux très élevé de défruitement; Minkley et al. (2006) ont donné une analyse très complète de cet accident. L'effondrement de 1873 à Varangéville n'est clairement pas associé à l'éclatement des piliers : le gros pilier central a un élancement beaucoup trop faible ; d'ailleurs les petits piliers carrés étaient fendus après l'accident mais avaient conservé leur hauteur initiale.

\section{2.}

\section{Rupture d'une couche raide du toit}

Une troisième possibilité de rupture brutale est la flexion et la rupture d'une couche (c compétente ) dans le toit immédiat. Dans la région de Stassfurt (SaxeAnhalt) c'est l'existence de la couche dite (c anhydrite principale » qui explique le caractère dynamique des effondrements (Menzel et Minkley, 1996). On peut être tenté de trouver dans un tel mécanisme une explication de l'effondrement de 1873 du quartier Saint-Maximilien. En effet, des centaines de cavités ont été lessivées dans la partie supérieure de la formation du Keuper inférieur dans laquelle la mine de Varangéville est ouverte. La formation de cratères à la surface du sol 
survient par effondrement de ces cavités quand elles sont agrandies jusqu'à ce que leur diamètre devienne égal aux deux tiers environ de leur profondeur (typiquement $130 \mathrm{~m}$ pour $200 \mathrm{~m}$ ). Des descriptions très précises peuvent être trouvées dans Buffet (1998) et Jeanneau (2005). Dans chacun de ces deux cas, à la fin du lessivage de la caverne, le toit de la cavité avait atteint le sommet de la formation salifère, à un peu plus de $200 \mathrm{~m}$ de profondeur. La couche marneuse surmontant le sel est alors dégagée; elle se dégrade progressivement pour tomber au fond de la caverne. Le processus se poursuit de proche en proche pendant plusieurs années, de manière irrégulière, par chute de couches de marnes et d'anhydrite. Boidin (2007) a analysé cette évolution: le toit de la caverne, lorsqu'il est remonté d'une cincuantaine de mètres, se trouve au contact d'une couche raide et rigide de 6 à $8 \mathrm{~m}$ d'épaisseur, la dolomie de Beaumont, à $145 \mathrm{~m}$ de profondeur. Cette couche finit par porter l'essentiel du poids du recouvrement et rompre (Klein et al., 2008), sans doute en raison des tractions engendrées par sa mise en flexion. Un mécanisme analogue d'effondrement a été décrit par Rothenburg et al., 2002, dans le cas de cavités de dissolution dans la région de Windsor-Detroit, à la frontière des USA et du Canada. Dans le cas de SaintMaximilien, la dolomie de Beaumont paraît trop peu profonde pour que ce mécanisme ait pu jouer un rôle (une douzaine de mètres sous la surface du sol, donc $140 \mathrm{~m}$ au-dessus du toit de la mine). A cette profondeur, la dolomie est souvent altérée par l'action des eaux souterraines et ne peut être considérée comme une couche compétente. Significativement, la formation de discontinuités verticales ( ( marche d'escalier ») n'a pas été observée après l'effondrement, au contraire du cas de la formation d'un cratère.

\section{3.t.}

\section{Les quartiers exploités ultérieurement}

La comparaison avec les quartiers voisins est plus instructive. Après l'accident de 1873, les méthodes d'exploitation de la mine de Varangéville furent profondément modifiées. Une plaque de sel de $20 \mathrm{~cm}$ d'épaisseur fut laissée au mur et on n'utilisa plus l'eau douce pour pré-découper le front, deux mesures qui contribuèrent à protéger les marnes du mur de l'action de l'air, de l'eau ou de la saumure. On conserva une hauteur de galerie de $5,5 \mathrm{~m}$, mais le taux de défruitement fut progressivement réduit ; $\tau=75 \%$ à la fin $\mathrm{du}$ XIX siècle, puis $60 \%$ et finalement $52 \%$ dans les quartiers actuels. On n'a observé dans ces quartiers, dont certains ont plus d'un siècle d'âge, que des désordres mineurs (chutes locales du toit, au croisement de galeries, écaillage local de piliers, soufflage du mur - sans doute d'ailleurs associé au flambage de la plaque de sel laissée au mur plutôt qu'à une montée des marnes du mur). Autrement dit, sans dégradation de la résistance mécanique des marnes du mur, il n'y a pas d'effondrement brutal.

\section{5}

\section{Le cas de la mine Dieuze}

La mine de Dieuze (Feuga, 2002), creusée à quel110 ques dizaines de kilomètres de Varangéville, mais un aussi le mur marneux à découvert, avec un taux de défruitement analogue. Elle a été envahie par de la saumure saturée en 1864 mais est restée parfaitement stable depuis, comme l'a montré un relevé de forme par sonar réalisé par Geoderis en 2002. Trois explications de ce bon état, inattendu compte tenu de l'expérience de Saint-Maximilien, ont été avancées. Elles ne sont d'ailleurs pas contradictoires:

- les marnes du mur immédiat, qui contient du sel, ne seraient pas dégradées par la saumure saturée ;

- à Dieuze la première couche de marnes, plus dure, n'a pas été entaillée par des rigoles et elle aurait protégé la couche sous-jacente de marnes brun-chocolat; - enfin cette mine, moins large, moins profonde, soutenue par la pression de la saumure après l'ennoyage, serait mécaniquement plus stable que Saint-Maximilien (Feuga et al., 2008). Cette dernière explication est la moins hypothétique, ce qui ne signifie pas que les deux précédentes soient inexactes.

\section{6he}

\section{Caractère unique de l'effondrement de Saint-Maximilien}

En conclusion, l'effondrement de Saint-Maximilien présente des caractéristiques uniques pour une mine de sel; ce n'est pas la rupture des piliers de sel, ni la rupture d'une couche raide dans le toit de la mine, mais le poinçonnement des marnes du mur par les piliers de sel de la mine qui est à l'origine de l'effondrement.

Il faut toutefois un second ingrédient pour expliquer la brutalité de l'effondrement; on a vu que les marnes humides sont viscoplastiques, et on pourrait imaginer une descente graduelle du toit qui suivrait souplement l'enfoncement progressif des piliers dans le mur. En fait c'est la raideur du toit de sel qui retarde longtemps une telle descente progressive du toit : le mur n'est rapidement capable que de porter une fraction du poids du recouvrement, mais le toit raide reporte sur les bords fermes l'excès de charge. Un modèle très simple montre que ce report ne peut être indéfini et qu'il existe une taille de la mine pour laquelle le report devient impossible sans rupture. La mine est à la profondeur $\mathrm{H}$; le poids par unité de surface de la colonne de terrains de recouvrement (contrainte géostatique) est $\mathrm{P}_{\infty}=\gamma \mathrm{H}$. Le contour de la mine est assimilé à un cercle de rayon $\mathrm{R}$. Le mur possède une résistance limitée, par exemple une cohésion $\mathrm{C}$ et un angle de frottement nul. La capacité portante du mur poinçonné par un pilier, soit $\omega \mathrm{C}$, est alors proportionnelle à la cohésion $\mathrm{C}$; la constante $\omega$ dépend notamment de la forme du pilier. Les piliers ne peuvent alors apporter au recouvrement, en moyenne, un support supérieur à $(1-\tau) \omega C$. L'équation d'équilibre des terrains, projetée sur la verticale, donne :

$$
\gamma H \pi R^{2}-(1-\tau) \omega C \pi R^{2}+2 \pi R H \bar{\sigma}_{\gamma z}
$$

(Fig. 5) où $\bar{\sigma}_{x,}$ est la valeur moyenne du cisaillement vertical qui s'applique le long de la surface verticale tracée à l'aplomb du contour du quartier. Lorsque le quartier s'élargit, ce cisaillement moyen croît comme le rayon $R$. Or le cisaillement moyen supportable est borné : le sel du recouvrement vérifie un critère de dilatance, qualitativement comparable à un critère de Coulomb, ou de Hoek and Brown, au-delà duquel sa résistance diminue. Comme la contrainte moyenne ne peut prendre que des valeurs limitées, de l'ordre de grandeur 
de $\left|I_{j}\right| / 3=\gamma z$, il existe une valeur du rapport $\mathrm{R} / \mathrm{H}$ pour laquelle l'équilibre du recouvrement est impossible ; des déformations localisées intenses apparaissent sur le pourtour de la mine; elles confèrent brusquement au toit une grande souplesse, qui autorise l'achèvement brutal du processus de poinçonnement jusque-là retenu par la présence d'un toit raide.

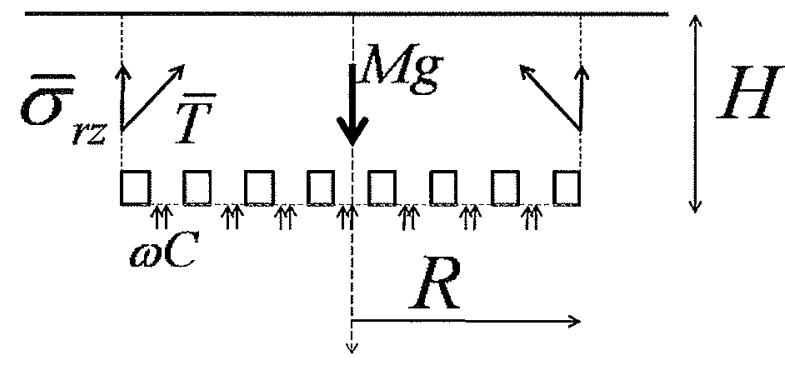

Hc. 5 Le poids du cylindre de terrains au-dessus de la mine ( $\left.M g=\rho \gamma \pi R^{2} H\right)$ est, au plus, équilibré par la somme de la capacité portante du mur sous les piliers $(\omega \mathrm{C} \times(1-$ $\tau) \pi R^{2}$ ) et de la résultante des cisaillements verticaux sur le contour $\left(\bar{\sigma}_{2} \times 2 \pi \mathrm{RH}\right)$.

The weight of the cylinder above the mine $\left(\mathrm{Mgg}=\rho \gamma \pi \mathrm{R}^{2} \mathrm{H}\right)$ is balanced by the sum of the bearing capacity of the marls layer beneath the pillars $\left(\omega \mathrm{C} \times(1-\tau) \pi \mathrm{R}^{2}\right)$ plus the vertical shear stresses which apply on the mine contour $\left(\overline{\mathrm{o}}_{\mathrm{z}} \times 2 \pi \mathrm{RH}\right)$

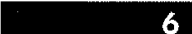

\section{Une reconstitution de l'évolution du quartier}

On peut ainsi esquisser une histoire mécanique du quartier. Le mur marneux était un point faible - d'autant plus que l'eau utilisée pour le pré-découpage s'infiltrait et réduisait sa résistance. L'ouverture progressive des chambres reportait sur les piliers une proportion initialement croissante du poids du recouvrement et créait des surfaces libres pour l'écoulement des marnes, permettant un début de poinçonnement du mur. Tant que les dimensions horizontales du quartier restaient faibles, le toit conservait une raideur globale suffisante pour empêcher qu'une flèche importante ne soit prise : le poinçonnement restait insensible. Au fil des années, le quartier s'élargissant, le toit devenait beaucoup plus souple; en même temps plus d'eau ou de saumure s'infiltraient dans le mur. Les deux phénomènes permettaient un poinçonnement accru, dont les effets devenaient perceptibles au voisinage du pilier central : la hauteur des galeries y diminuait. Paradoxalement, la charge sur les piliers tendait à décroître, puisqu'elle ne peut pas excéder de beaucoup la capacité portante du mur, et une large fraction du poids du recouvrement se reportait sur les bords fermes. A la verticale du pourtour extérieur de la mine, des contraintes de cisaillement intenses se développaient dans le toit de sel où le critère de dilatance était atteint dans une zone d'extension verticale croissante. Le sel s'y radoucissait et, à grande échelle, le toit de sel, dont la raideur et la résistance avaient limité jusque-là le poinçonnement du mur, s'assouplissait alors brutalement, permettant en quelques secondes le poinçonnement complet par le pilier central du mur affaibli par l'infiltration de l'eau.
Ce schéma, qui ne s'appuie que sur des considérations élémentaires d'équilibre, fournit le mécanisme de l'effondrement. Toutefois pour que ce schéma soit crédible, on doit vérifier qu'il est compatible avec ce qu'on sait du comportement rhéologique des marnes humides, du sel et du toit; et qu'on peut rendre compte quantitativement des traits principaux du comportement du quartier pendant ses 17 années d'existence.

\section{Comportement rhéologique des diverses parties de la mine}

\section{2.}

\section{Comportement des piliers de sel}

Paradoxalement, le comportement rhéologique du sel gemme, malgré son caractère très particulier, ne joue pas (hormis la dilatance) un rôle essentiel dans l'accident. Le sel gemme est un matériau viscoélastique non linéaire : il flue même sous charge très faible. A Saint-Maximilien la profondeur du quartier est $\mathrm{H}=156 \mathrm{~m}$, et la contrainte géostatique est donc modérée, de l'ordre de $\mathrm{P}_{\infty}=\gamma \mathrm{H}=3,5 \mathrm{MPa}$. Dans un quartier peu étendu on peut donc attendre des contraintes déviatoriques - donc des vitesses de déformation modérées, sauf peut-être dans les piliers eux-mêmes. En effet le taux de défruitement $\tau$ étant supérieur à $80 \%$, la charge tributaire est au moins de $q=y \mathrm{H} /$ $(1-\tau) \approx 17,5 \mathrm{MPa}$, une valeur susceptible d'engendrer un fluage rapide des piliers. En fait les valeurs réelles étaient sans doute bien plus faibles - les mineurs n'ont décrit aucune manifestation caractéristique d'une déformation intense des piliers, même dans les zones ouvertes depuis 17 ans. La raison en est que les piliers n'appliquent rapidement sur le mur guère plus que sa capacité portante, que l'on évalue plus loin. De ce fait le comportement viscoplastique du sel ne joue qu'un rôle relativement discret du point de vue du mécanisme d'effondrement; les piliers sont presque rigides en comparaison des marnes sur lesquelles ils s'appuient. Pour les calculs, on a utilisé une description du sel de Varangéville comme un matériau de Lemaitre-MenzelSchreiner proposée par Vouille (1986).

Le critère de dilatance (ou radoucissement) du sel joue pour sa part un rôle essentiel ; il est abordé par la suite.

\section{2}

\section{Comportement des marnes}

\section{1.}

\section{Les marnes dans les formations salifères}

La mine de Varangéville est exploitée depuis plus d'un siècle et les mineurs ont observé depuis toujours que les marnes du mur se dégradent rapidement au contact de l'eau et/ou de la saumure. Ce phénomène est aussi observé dans les marnes des formations salifères en couches exploitées par dissolution. Par exemple l'étude des cavités de Hengelo aux Pays-Bas conduit Bekendam (2000) à détailler les mécanismes physicochimiques qui conduisent à cette dégradation. Les 
marnes de Varangéville ont été étudiées d'un point de vue géomécanique par Vouille (1986) qui a mis en œuvre différents essais. Le prélèvement des échantillons était déjà difficile, et les résultats des essais étaient dispersés, de sorte que la définition d'un critère de résistance à court terme n'était pas aisée. Pour les marnes sèches et peu profondes (c'est-à-dire prélevées dans le mur immédiat) Vouille (1986) propose un critère de Tresca $\mathrm{C}=2 \mathrm{MPa}, \varphi=0^{\circ}$ tout en considérant ces valeurs comme très pessimistes (plusieurs techniques de détermination ont été utilisées, et certaines conduisent à proposer un critère de Mohr-Coulomb). Des éprouvettes étaient soumises à une charge constante modérée (de 2 à $6 \mathrm{MPa}$ ) puis mises en contact après quelques jours avec de la saumure saturée : la rupture survenait après quelques jours, ou même immédiatement. Plus récemment Boidin (2007) a réalisé sur des marnes de la même formation géologique des essais qui suggèrent fortement que c'est l'hydratation des particules d'anhydrite disséminées au sein des marnes - un mécanisme déjà proposé par Braconnier il y a 130 ans - qui explique la dégradation des matériaux, plutôt que le gonflement des smectites de la phase argileuse, mécanisme qui est inhibé en présence de saumure saturée.

\section{5. \\ Infiltration de l'eau dans le mur de la mine}

Il est difficile d'évaluer l'étendue que la zone dégradée par l'action de l'eau au mur de la mine avait atteinte à la veille de l'accident de 1873. Depuis l'accident on n'utilise plus d'eau dans la mine, de sorte qu'on manque de données et d'observations. Néanmoins il est certain que, par endroits, l'eau pouvait pénétrer profondément dans le mur, sous la couche supérieure dure anhydritique, jusqu'aux marnes brun-chocolat qui sont moins dures et particulièrement sensibles à l'action de la saumure.

« Une autre circonstance a facilité l'amollissement de cette marne. En raison de l'inclinaison générale de $12 \mathrm{~mm}$ par mètre de la $11^{\mathrm{e}}$ couche vers le nord nordouest, il a fallu pratiquer dans la marne des rigoles dont la profondeur atteignait 2,50 $\mathrm{m}$ en certains points. Ajoutons enfin que sa machine à colonne d'eau et son bassin étaient placés dans une cavité creusée dans la marne, en partie sous les gros piliers du puits $n^{\circ} 1 \ldots$ ) (Braconnier, 1873c, p. 1).

On peut en déduire que le mur dans la partie centrale de la mine, au voisinage du pilier central, où l'on collectait les saumures produites, était particulièrement affecté :

( L'amollissement de la marne était encore facilité par les nombreuses excavations qui y étaient pratiquées [...]. C'est dans le voisinage du puits que ces excavations étaient surtout nombreuses et profondes ; c'est là aussi que la marne était soumise, depuis le plus longtemps, à l'influence de l'air et de l'eau. C'est donc là que sa cohésion avait disparu le plus complètement et sur la plus grande profondeur » (Anonyme, 1873, p. 625).

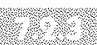

\section{Poinçonnement du mur marneux par les piliers}

Quand elles sont détrempées, les marnes du mur présentent une cohésion faible. Par contraste, les piliers de sel, qui sont peu chargés, comme on le vérifie a posteriori, fluent lentement et peuvent être considérés comme rigides: l'enfoncement des piliers dans le mur peut être approché par le problème du poinçon rigide appliqué sur un demi-espace (visco-)plastique.

Pour calculer sa capacité portante, on a supposé que le mur marneux était constitué d'un matériau purement cohérent (pas d'angle de frottement) mais qu'il présentait une viscosité (matériau de Bingham). Outre que la viscosité régularise les calculs, on voulait décrire la cinématique de l'évolution, depuis l'ouverture de la mine jusqu'à l'effondrement. Pour un tel matériau, l'écoulement libre apparaît pour une valeur déterminée $\omega=q / \mathrm{C} d u$ rapport entre la charge et la cohésion. Cette valeur est indépendante des caractéristiques élastiques et visqueuses du matériau et des dimensions du pilier; elle dépend de la forme du pilier. Le cas du pilier cylindrique posé sur un demi-espace infini a été traité semi-analytiquement par Salençon et Matar (1982). Le rapport critique vaut environ $\omega=q / C \approx 6$. On a conduit des calculs numériques pour le cas des piliers minces toriques (les calculs numériques de l'évolution de la mine seront conduits avec l'hypothèse d'axisymétrie) ; une valeur comparable a été trouvée.

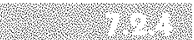 \\ Choix d'un comportement rhéologique des marnes du mur}

Ces ordres de grandeur constituent un guide utile. Certains quartiers de la mine actuelle (le mur y est sec) ont été exploités avec un taux de défruitement de $\tau=75 \%$ et un recouvrement localement égal à $\mathrm{H}=200 \mathrm{~m}$. On n'y a observé aucun signe de poinçonnement. Leur extension horizontale est suffisante pour que l'hypothèse de la charge tributaire y soit raisonnable, ce qui conduit à une estimation de $q=\bar{\sigma}_{z z}=\gamma \mathrm{H}$ / $(1-\tau)=17,5 \mathrm{MPa}$ environ pour la contrainte verticale moyenne sur les piliers, de sorte que la cohésion des marnes sèches est supérieure à $\mathrm{C}=\mathrm{q} / \omega \approx 3 \mathrm{MPa}$ (par ailleurs, l'existence d'un angle de frottement non nul est probable). Les marnes humides de Saint-Maximilien avaient manifestement une cohésion bien plus faible, mais celle-ci ne peut être estimée que par tâtonnements en effectuant le calcul complet de l'évolution de la mine et en cherchant à rester le plus proche des observations faites en 1873, notamment celles qui concernent les déplacements. On a essayé plusieurs valeurs et, comme indiqué plus loin, on a trouvé que $\mathrm{C}=0,75 \mathrm{MPa}$ donnait un bon accord avec les observations faites en place.

Il serait certainement plus exact de faire dépendre la cohésion de la profondeur, de la distance au puits $n^{\circ} 1$ et du temps, car la dégradation des marnes par l'eau n'a été ni uniforme ni instantanée. Mais, aucune description crédible du processus de dégradation n'étant disponible, on a jugé préférable de donner à la cohésion une valeur faible mais homogène - rendre le mur moins résistant sous le seul pilier central aurait pu par exemple sembler une façon de préjuger du mécanisme d'effondrement.

La viscosité était encore plus difficile à choisir. La viscosité du sel à température ambiante sous faible charge est de l'ordre de $\mu=10^{17} \mathrm{~Pa} . \mathrm{s}$ (Bérest et al., 2005) et il fallait manifestement adopter une valeur plus basse pour les marnes qui se sonl déformées bien plus vite que les piliers de sel. On a retenu $\mu=10^{15}$ Pa.s ; des valeurs plus faibles encore ne changeaient pas qualitativement les résultats mais rendaient les calculs 
très longs. Après la présente étude, il apparut que la valeur choisie n'était pas en désaccord avec les résultats d'essais de laboratoire postérieurs décrits par Boidin (2007).

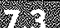

\section{Comportement du toit}

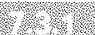

\section{Raideur du sel et raideur du toit de sel}

Le choix d'une cohésion faible des marnes du mur dégradées par l'eau implique que leur poinçonnement par les piliers peut survenir très tôt. Si le toit était extrêmement souple, la mine se fermerait complètement avant que l'effondrement ne devienne possible. S'il était extrêmement raide, on n'observerait guère de subsidence ni de convergence avant l'effondrement : le poinçonnement commencerait mais serait rapidement bloqué, seule une flèche très limitée du toit étant possible. On dispose de ce point de vue de quelques observations précieuses de Braconnier (voir paragraphe 4.1) qui établissent qu'à la veille de l'accident la subsidence était décimétrique et que près du pilier central la hauteur d'une galerie s'était réduite de $80 \mathrm{~cm}$ au moins.

La raideur globale du toit est une notion différente du module de la roche qui le constitue. En fait, si on assimile le sel au-dessus du quartier (sans pilier) à une plaque élastique circulaire encastrée de rayon $R$, de flèche $u=u(r)$, supportant une surcharge uniforme $p$, on peut définir son module global comme :

$$
\frac{p}{u(0)}=\frac{64 E I}{R^{4}}
$$

Le toit devient donc beaucoup plus souple quand la mine s'élargit. L'inertie à la flexion I n'est pas facile à apprécier ; elle vaut en principe $I=\eta^{3} / 12$ pour une couche unique d'épaisseur $\eta$ petite devant R. En fait il est fréquent qu'un toit soit composé d'une succession de couches raides séparées les unes des autres par des couches plus molles (argiles, marnes) qui n'offrent qu'une résistance très faible et permettent un glissement relatif aisé des couches raides les unes par rapport aux autres. Supposons que l'épaisseur totale soit composée de $\mathrm{N}$ couches raides d'épaisseur $\eta / \mathrm{N}$ séparées par des interlits très minces et très peu résistants. L'inertie globale est alors la somme des inerties des $N$ couches, I(N) $=\mathrm{N} \times \eta^{3 / 12 \mathrm{~N}^{3}}=\mathrm{I}(1) / \mathrm{N}^{2}$, donc bien plus faible que l'inertie d'une couche unique d'épaisseur $\eta$. Pour grossier qu'il soit, ce modèle montre que la raideur globale à la flexion d'un toit peut être beaucoup plus faible que l'impression tirée de la rigidité d'une éprouvette ne le suggère.

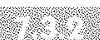

\section{Les discontinuités du toit à Varangéville}

La formation salifère contient un grand nombre de couches horizontales qui ont été décrites par les géologues (par exemple Marchal, 1983). La note aux Annales des mines (Anonyme, 1873) répertorie 10 couches marneuses qui séparent les 11 couches de sel entre le toit du sel et le niveau de la mine ; leur épaisseur varie de $0,5 \mathrm{~m}$ à $3 \mathrm{~m}$. Braconnier (1873c) souligne, après sa seconde visite, que plusieurs minces couches marneu- ses contenues dans le toit immédiat de la mine ont joué un rôle mécanique manifeste, puisque des fractures horizontales s'y sont développées pendant l'effondrement ; ces fractures, entre couches décollées du toit, sont bien visibles sur la figure 4. Plus inattendues dans une formation de sel en couche, on observe aussi, à la fois en $4^{\mathrm{e}}$ et $11^{\mathrm{e}}$ couches, de nombreuses discontinuités verticales, remplies de sel, de marnes et d'anhydrite. Le tracé d'au moins certaines d'entre elles est reporté sur le plan de la mine de la figure 1. Une description détaillée de ces fractures verticales, organisées suivant un réseau polygonal, est donnée par Robelin et Bonijoly (1990). Avant l'effondrement, on avait observé que « Un grand nombre de piliers carrés présentaient sur toute leur hauteur des fentes atteignant jusqu'à $5 \mathrm{~cm}$ [...] précisément dans les veines presque verticales de marnes qui traversent irrégulièrement la onzième couche s) (Braconnier 1873c, p. 2). Dans la suite on montre que ces fractures ont été ouvertes pendant l'exploitation par les fortes contraintes horizontales engendrées par l'écoulement des marnes sous les piliers, lorsque ceux-ci ont commencé à poinçonner le mur.

Pour la modélisation numérique, une prise en compte détaillée de toutes ces discontinuités était évidemment hors d'atteinte ; une description simplifiée était nécessaire. Un modèle élastique orthotrope de révolution était une option intéressante, mais on a adopté une solution plus grossière en intercalant trois couches horizontales de ( marnes ) de 3 mètres d'épaisseur dans la masse de sel. Un plus grand nombre d'intercalations eut été plus réaliste, mais aurait conduit à un maillage très lourd, compte tenu du grand nombre de calculs nécessaires. On a donc complété ( l'assouplissement ) du toit en réduisant substantiellement la raideur du sel (et des marnes intercalées), avec l'inconvénient de mal respecter la raideur vraie pour les transformations autres que la flexion d'ensemble du toit.

\section{1)}

\section{Un modèle du toit}

Pour estimer la raideur globale du toit ainsi constitué, on a effectué des calculs numériques (le détail du modèle est donné au paragraphe suivant) pour une ( caverne ) cylindrique de $160 \mathrm{~m}$ de rayon à $160 \mathrm{~m}$ de profondeur (c'est-à-dire sans piliers; il est probable qu'une telle caverne ne serait pas stable, mais c'est la raideur du toit qui nous intéresse ici, et non pas sa résistance). On a examiné trois hypothèses:

(1) le toit est (c mou ) (le module du sel est $E=1 \mathrm{GPa}$ et celui des marnes est $E^{\prime}=0,5 \mathrm{GPa}$ );

(2) le toit est ( raide $)\left(E=5 \mathrm{GPa}\right.$ et $\left.E^{\prime}=1 \mathrm{GPa}\right)$ et

(3) le toit est ( très raide ) $(E=26 \mathrm{GPa}$ et il n'y a pas d'intercalations de marnes).

La flèche maximale du toit, prise au centre du quar tier, était respectivement $74 \mathrm{~cm}, 28 \mathrm{~cm}$ et $10 \mathrm{~cm}$. Il était clair que seul le toit “ mou » pouvait pousser suffisamment les piliers dans le mur avant l'effondrement et rendre ainsi compte des convergences observées près du pilier central. Les calculs conduits ultérieurement (pour la mine complète avec ses piliers) montrent que la subsidence calculée avec l'hypothèse du toit ( mou » était de $32 \mathrm{~cm}$ en 1873 (juste avant l'effondrement) et beaucoup moins avec les deux autres modèles et que le déplacement vertical du pilier central à la même période était de $40 \mathrm{~cm}$, alors que le mur était remonté de $50 \mathrm{~cm}$, d'après 
les calculs, dans la galerie contiguë au pilier central, soit une convergence apparente de $90 \mathrm{~cm}$, compatible avec les observations de l'époque, et qui donne à l'hypothèse ( molle ) une crédibilité. Néanmoins le modèle du toit, s'il fournit une description assez réaliste de la flexion du toit, ne peut avoir l'ambition de décrire le détail de son comportement mécanique.

\section{8}

\section{Calcul de l'évolution de la mine}

\section{8.}

\section{Le modèle}

Le quartier réel comporte un pilier central de $29 \mathrm{~m} \times 40 \mathrm{~m}$ et un grand nombre de petits piliers de $6 \mathrm{~m} \times 6 \mathrm{~m}$ (Fig. 1). Le contour extérieur du quartier est approximativement un cercle de $160 \mathrm{~m}$ de côté. L'attention ne se porte pas sur le comportement individuel de chaque pilier - ce ne sont pas eux qui se sont rompus - mais plutôt sur le comportement d'ensemble du quartier, de sorte qu'on a adopté une description axisymétrique simplifiée. Une contrainte importante était de respecter le taux de défruitement de $\tau=82 \%$. Les centaines de piliers de la mine réelle ont donc été remplacés par 8 (" piliers ) toriques de $2,87 \mathrm{~m}$ de largeur délimitant 9 (c chambres » de 13,18 m de largeur, numérotées de 1 à 9 du centre du quartier vers l'extérieur. Le pilier rectangulaire central était remplacé par un pilier circulaire de rayon $21,37 \mathrm{~m}$ (cette description du quartier, et celle de la chronologie du creusement sont dues à Gérard Vouille, que les auteurs remercient). Le modèle complet du massif est un cylindre de $400 \mathrm{~m}$ d'épaisseur et $300 \mathrm{~m}$ de rayon. Sur sa base les déplacements verticaux et les cisaillements horizontaux sont nuls. Sur les côtés la contrainte géostatique est imposée. Les éléments sont des triangles à 6 nouds; dans la plupart des calculs on utilise 52548 éléments (105 434 noeuds).
Le calcul numérique permet d'analyser les effets de la viscosité du sel et des marnes. Il faut donc tenir compte de la chronologie du creusement du quartier. Il est resté ouvert de 1855 (après le creusement du puits $n^{\circ}$ 1) à 1873 (date de l'effondrement). On a supposé qu'entre ces cleux dates le tonnage annuel de sel extrait était constant, ce qui fixe la chronologie de l'ouverture des 9 chambres du modèle, le creusement de la dernière commençant peu après 12 ans et s'achevant à la quinzième année. On poursuit les calculs pendant 10 années supplémentaires (la mine réelle est alors effondrée) pour examiner les tendances de l'évolution.

\section{9}

\section{Évaluation des contraintes}

Quand on ouvre les premières chambres, au voisinage du pilier central, la charge verticale portée par les piliers créés (dont le pilier central) croît depuis la pression géostatique $-\gamma \mathrm{H}=3,5 \mathrm{MPa}$ à une profondeur de $150 \mathrm{~m}$ - jusqu'à une valeur plus grande qui est notamment influencée par le rayon atteint par le quartier. Dès lors que la cohésion sélectionnée pour les marnes du mur est faible, et que le toit modélisé est « mou », la capacité portante du mur (qui vaut 6 à 7 fois la cohésion) est rapidement dépassée et les piliers, dont le pilier central, commencent à poinçonner le mur. Chaque fois qu'une nouvelle chambre torique est ajoutée, un surcroît de charge s'applique immédiatement sur les piliers déjà créés (Fig. 6; la charge représentée est la charge additionnelle, qu'on doit ajouter à la pression géostatique initiale) mais il diminue rapidement : la viscosité des marnes du mur n'est pas considérable et permet une résorption relativement rapide de l'écart à la capacité portante (Fig. 6). La charge sur le pilier central reste toujours plus faible que celle qui s'exerce sur les petits piliers toriques; inversement la contrainte verticale additionnelle sur le bord ferme - moyennée jusqu'à la limite extérieure du modèle - ne cesse de croitre.

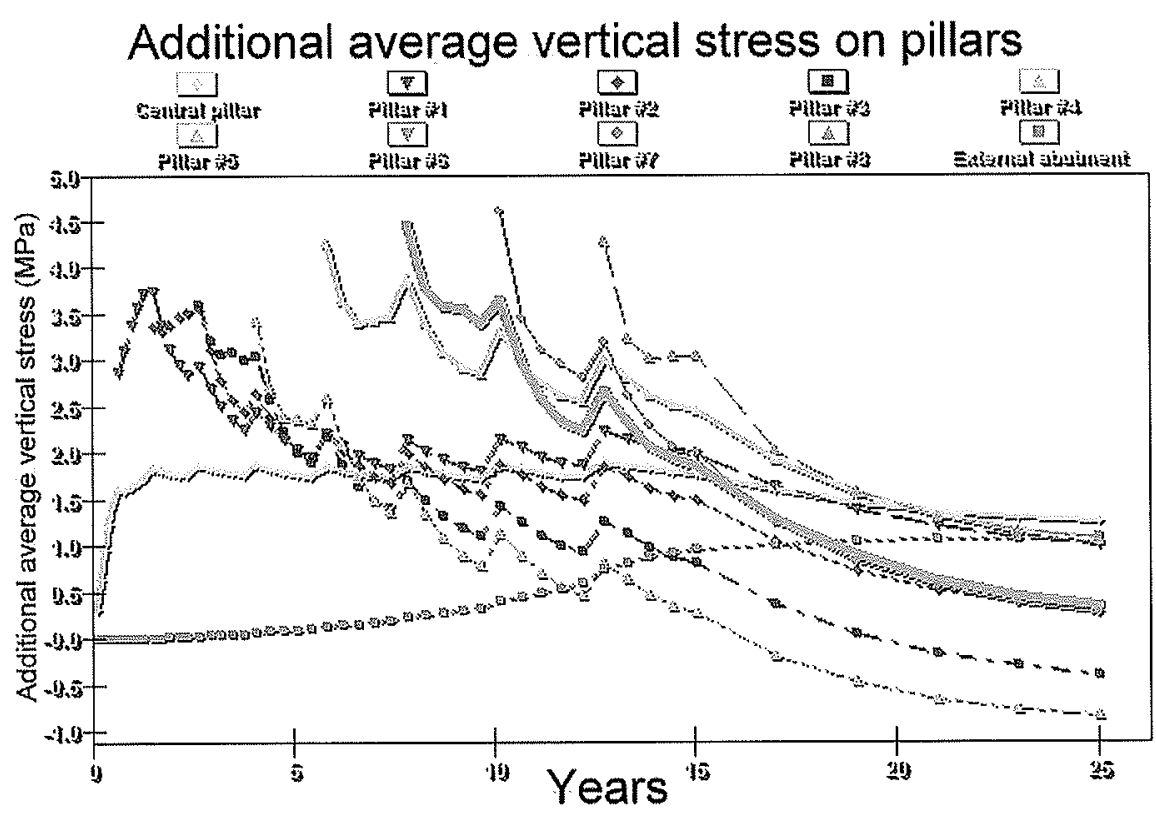

HG. 6 Charges verticales additionnelles supportées par les huit piliers du modèle et le bord ferme (contour extérieur de la mine) en fonction du temps après l'ouverture de la mine.

Additional vertical loads on the pillars and external abutment as a function of time. 


\section{Déplacements}

Les piliers pénètrent dans les marnes sous-jacentes qui sont repoussées vers les surfaces libres, c'est-àdire les murs des deux galeries voisines, qui se soulèvent. Comme le volume des marnes demeure constant, le rapport entre l'enfoncement des piliers - qui est à peu près égal à la descente du toit - et le soulèvement du mur est dans le rapport des aires horizontales des piliers et des chambres, $\tau /(1-\tau) \approx 4$, sauf toutefois au voisinage du pilier central et des bords fermes, où ce rapport est localement plus important. Notamment le poinçonnement précoce du mur par le pilier central déplace un volume important de marnes, permettant le soulèvement du mur de la galerie qui lui est contiguë. La figure 7 donne une image plus détaillée des déplacements dans les deux premières galeries les plus proches du pilier central. La descente du toit $(40 \mathrm{~cm})$ est identique dans les deux galeries. Les piliers poinçonnent le mur, de $38 \mathrm{~cm}$ pour le pilier central, et le mur se soulève de $40 \mathrm{~cm}$ dans la galerie $\mathrm{n}^{\circ} 1$ (le taux de défruitement local est proche de $50 \%$, et la descente du toit est presque égale à la montée du mur), moins dans les autres galeries, où le soulèvement est plus marqué au voisinage des piliers. La réduction de hauteur des galeries est la quantité que les mineurs pouvaient observer : le calcul la prédit un peu supérieure à $80 \mathrm{~cm}$ dans la galerie $n^{\circ} 1: c^{\prime} e s t l^{\prime}$ ordre de grandeur de ce qui a été rapporté pour la galerie Sainte-Julie. Le modèle prédit aussi que le bord ferme poinçonne les marnes: cet effet n'est pas très réaliste, les marnes étant vraisemblablement restées sèches dans cette zone, mais le modèle ne rend pas compte de cette hétérogénéité probable de la cohésion.

Sous le premier pilier, à $35 \mathrm{~m}$ environ de l'axe du puits, l'écoulement des marnes est à peu près symétrique, de sorte qu'à la base du pilier des cisaillements de signe opposé sont appliqués sur la moitié gauche et la moitié droite. Le pilier étant raide en comparaison des marnes, des tractions horizontales apparaissent dans sa partie inférieure. Dans la vraie mine, ce mécanisme explique l'ouverture de fractures verticales, qui suivent les surfaces de faiblesse mécanique constituées par les fentes remplies de marnes qui traversent les piliers. Dans la chambre la plus extérieure (non représentée) des compressions sub-horizontales sont engendrées dans les coins supérieur et inférieur du côté du bord ferme, comme il est logique au voisinage de l'encastrement d'une plaque en flexion. Après l'effondrement, la flexion du toit est plus grande, et ces contraintes de compression ont dû atteindre des valeurs considérables qui expliquent les dégâts observés dans le toit. Toutes ces remarques sont qualitatives, le modèle numérique ne reflétant que grossièrement la géométrie réelle des piliers.

\section{.}

\section{Variantes}

Les mêmes calculs ont été conduits en conservant l'hypothèse du toit ( mou ) mais en augmentant la cohésion du mur. Pour des valeurs de $\mathrm{C}=1$ ou 1,25 $\mathrm{MPa}$ (au lieu de $\mathrm{C}=0,75 \mathrm{MPa}$ dans le cas de référence) l'évolution des charges portées par les piliers est la même que dans le cas de référence : elles convergent lentement vers la capacité portante du mur, qui est proportionnelle à sa cohésion. La réduction de hauteur de la première galerie après 25 ans est de 74 et $25 \mathrm{~cm}$, respectivement, au lieu de $105 \mathrm{~cm}$ dans le modèle de référence. Dans la réalité la convergence était certainement supérieure à $80 \mathrm{~cm}$ (après 15 ans) de sorte que $\mathrm{C}=0,75 \mathrm{MPa}$ apparaît comme le meilleur choix. Quand on retient $\mathrm{C}=2 \mathrm{MPa}$, la charge moyenne sur les piliers est de l'ordre de $9 \mathrm{MPa}$, moins que la capacité portante du mur qui est de l'ordre de $\omega \mathrm{C}=12 \mathrm{MPa}$, et les piliers ne poinçonnent pas le mur; d'ailleurs le déplacement vertical du toit après 25 ans n'est que de $35 \mathrm{~mm}$ : cette configuration est sans doute proche de celle de la mine sèche, telle qu'elle a été exploitée (sans eau) après 1873. Il est vraisemblable toutefois que les caractéristiques réelles des marnes sèches sont encore plus élevées (aucun indice de poinçonnement du mur n'a été observé depuis 135 ans dans la mine sèche).

On a aussi envisagé le cas d'un toit ( raide ) en conservant la cohésion de $\mathrm{C}=0,75 \mathrm{MPa}$. Les charges verticales à 25 ans sur les piliers sont à peu près

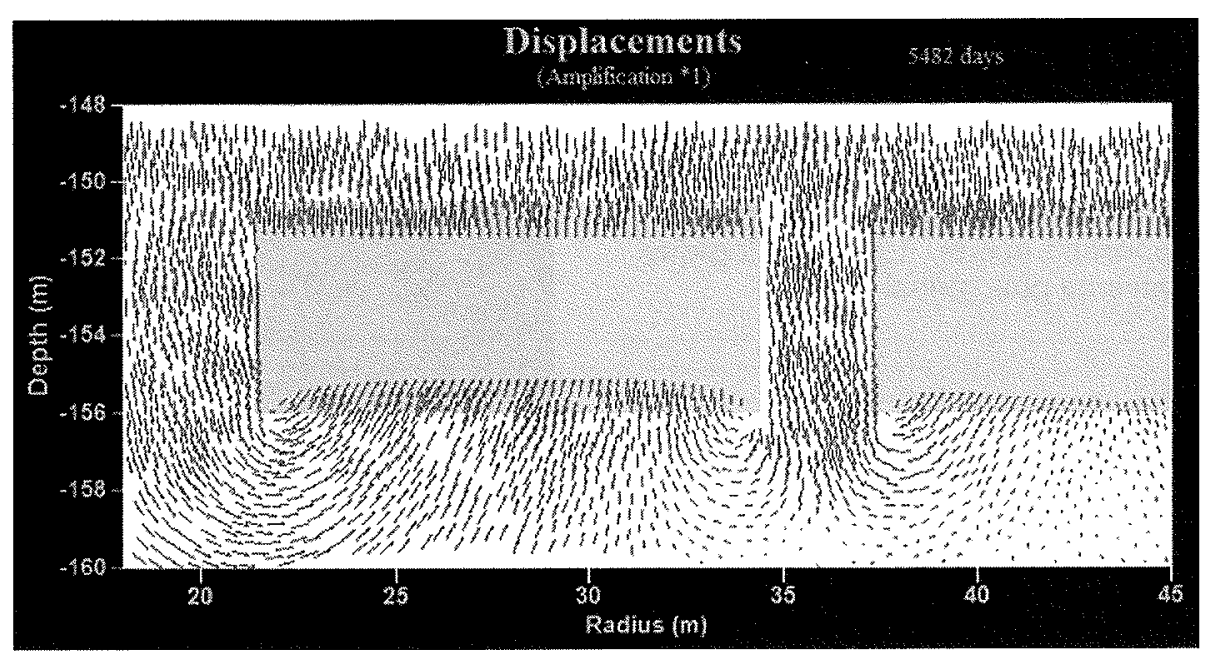

Fe. 7 Champ des déplacements dans les deux premières galeries 15 ans après l'ouverture de la mine.

Displacement field in the first and second galleries 15 years after mine creation. 
inchangées, un résultat logique puisque ces charges tendent à devenir proportionnelles à la cohésion des marnes du mur. Mais avant cette date elles sont plus élevées que dans le modèle de référence, le poinçonnement étant plus progressif : le déplacement vertical à la base du pilier central à 25 ans n'est cque de $21 \mathrm{~cm}$ au lieu de $44 \mathrm{~cm}$ dans le modèle de référence, qui doit donc être préféré.

\section{9}

\section{L'effondrement}

\section{9.}

\section{A la recherche d'un mécanisme de ruine}

Le modèle, tel que décrit jusqu'ici, ne contient pas de mécanisme pouvant conduire à la ruine brutale du quartier. Certes l'écoulement libre est rapidement atteint sous les piliers; mais le toit conserve une certaine raideur à la flexion qui limite le déplacement vertical des piliers et empêche un poinçonnement complet. Une ruine brutale ne peut qu'être associée à une chute brutale de la valeur d'un des deux paramètres essentiels, la cohésion du mur ou la raideur du toit, qui déterminent chacun un mécanisme particulier de rupture.

Une condition nécessaire pour que le premier mécanisme soit efficace est que le toit soit encore beaucoup moins raide qu'on ne l'a supposé jusqu'ici. On peut imaginer alors que la cohésion du mur marneux - qu'on a choisie jusqu'ici faible mais uniforme et constante dans le temps - chute brutalement dans une large zone au cours du processus de dégradation par l'eau. Ce n'est pas une hypothèse invraisemblable, mais elle n'est pas conforme à l'image progressive que l'on peut se faire de l'infiltration de l'eau dans le mur.

Dans le second mécanisme, c'est la raideur du toit qui chute brutalement. On a observé que dans la galerie la plus extérieure le toit s'est effondré sur une épaisseur de plus de 3 mètres, et on pourrait être tenté d'y voir l'origine au moins immédiate de l'effondrement; mais le toit de sel fait $70 \mathrm{~m}$ d'épaisseur, et une réduction de $3 / 70=4 \%$ de cette épaisseur ne suffit pas à réduire significativement la raideur à la flexion. La chute du toit dans les galeries extérieures est très vraisemblablement une conséquence plutôt qu'une cause de l'effondrement. D'ailleurs il est difficile de concevoir que cette chute du toit, si elle avait été l'élément déclencheur, n'eût pas été précédée de signes précurseurs : les mineurs n'en rapportent aucun. Il est plus logique de chercher un mécanisme plus global.

\section{3.}

\section{Dilatance au-dessus du pourtour}

On a vu que l'équilibre mécanique d'ensemble du quartier exige que des cisaillements verticaux croissants se développent sur le pourtour du cylindre constitué par les terrains au-dessus du quartier. Or on sait que le sel s'endommage (sans rompre franchement) quand l'état de contrainte sort d'un domaine dit (c dilatant ) (la formulation la plus simple du critère $11 \mathrm{C}_{\text {dilatant est } \sqrt{J}_{2}=C\left|I_{1}\right| ; I_{1}=\sigma_{\mathrm{kk}} \text { désigne le premier inva- }}^{\text {riant du tenseur des contraintes et } J_{2}=\mathrm{s}_{\mathrm{ij}} \mathrm{s}_{\mathrm{ji}} / 2 \text { où } \mathrm{s}_{\mathrm{ij}}=}$ $\sigma_{\mathrm{ij}}-\sigma_{k k} \delta_{\mathrm{ij}} / 3$ est le second invariant du tenseur déviateur des contraintes) à l'extérieur duquel une microfissuration se développe, accompagnée de l'augmentation de la perméabilité, de la chute de la vitesse du son, de l'accroissement de l'émission acoustique et de celui de la vitesse de déformation, décrit par Chan et al. (1996) sous la forme d'un endommagement au sens de Katchanov ou Lemaitre. La description du comportement post-dilatant d'une structure complexe est délicate - une localisation des déformations est probable - mais on peut en première approche utiliser une analogie : si une large zone " dilatante » (c'est-à-dire où le critère dilatant est excédé) s'étend et notamment traverse la masse de sel, l'équivalent d'un écoulement libre devient possible. On a donc tracé l'évolution au cours du temps de la zone où le critère dilatant est dépassé. On a utilisé le critère dilatant assez élaboré proposé par De Vries (2003) pour le sel en couches de Cayuta (état de New York); on a utilisé toutefois des valeurs des paramètres de ce modèle qui rendent le sel de Varangéville un peu moins résistant que celui de Cayuta. On désigne par FOS (Factor of Safety) la valeur du critère pour l'état de contraintes calculé (ce rapport est supérieur à 1 quand
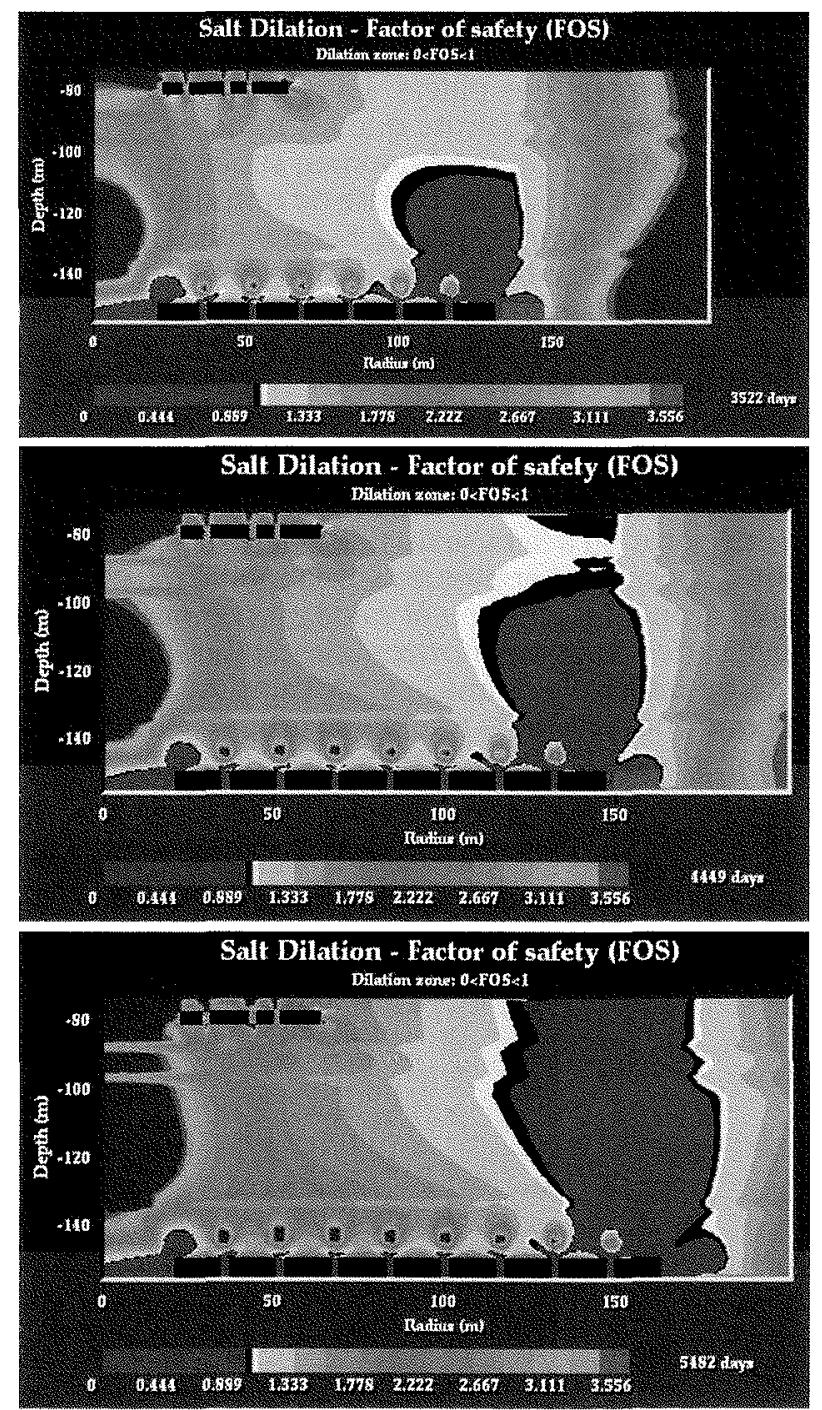

76. 8 Progression de la zone dans laquelle le critère dilatant est dépassé pendant l'excavation des galeries $n^{\text {os }} 7,8$ et 9 .

Progression of the zone in which the dilation criterion is met during excavation of galleries $n^{\text {os }} 7,8$ and 9 . 
la dilatance n'est pas atteinte). Les résultats des calculs, dans lesquels on a pris en compte l'existence de l'exploitation en $4^{\mathrm{e}}$ couche, sont présentés à la figure 8. Les trois dessins montrent le développement de la zone ( dilatante ), successivement après l'ouverture des $7^{\mathrm{e}}$, $8^{\mathrm{e}}$ et $9^{\mathrm{e}}$ galeries. Après que cette dernière est ouverte, s'étend du toit du quartier jusqu'au toit du sel une zone dilatante continue, qui peut fonctionner un peu comme une rotule plastique.

\section{0}

\section{Conclusion}

L'analyse des observations rapportées par les témoins de l'accident du quartier Saint-Maximilien permet de proposer une description cinématique de l'effondrement. L'origine de celui-ci doit être cherchée dans le poinçonnement par les piliers de sel des marnes du mur, affaiblies par la présence de grandes quantités d'eau utilisées pour l'exploitation de la mine. Le caractère brutal de l'effondrement, pour sa part, a pour origine vraisemblable une perte de résistance des terrains du recouvrement à l'aplomb du pourtour extérieur de la mine, qui était le siège de sollicitations croissantes quand la mine s'élargissait. On a pu rendre compte de l'effondrement par le calcul numérique en traitant les marnes du mur comme un milieu viscoplastique de Bingham de cohésion réduite, en dotant le toit en sel, qui est traversé par de nombreuses discontinuités horizontales, d'une raideur à la flexion faible, et en prenant en compte un critère dilatant du sel proche de ceux proposés dans la littérature. Ces caractéristiques font de l'effondrement du quartier Saint-Maximilien un exemple probablement unique dans l'histoire des mines de sel.

\footnotetext{
REMRRGIEMENTS

Cette étude a été rendue possible par un financement de Geoderis, l'agence de l'Etat chargée de l'Après-Mine, qui a autorisé la publication de cet article. La Compagnie des salins du Midi et des salines de l'Est (CSME) a aimablement autorisé la reproduction de photographies provenant de ses archives et Emmanuel Hertz a fait part aux auteurs de nombreuses remarques très précieuses. Gérard Vouille a proposé le découpage du creusement des chambres utilisé pour les calculs et rectifié plusieurs erreurs du manuscrit. Des discussions avec Jean Salençon et Alain Pecker ont permis d'éclaircir divers points relatifs à la capacité portante et aux effets dynamiques.
}

\section{Bibliographie}

Anonyme - Note sur l'effondrement de la mine de sel gemme de Varangéville Saint-Nicolas (Meurthe-et-Moselle). Annales des mines, $7^{\mathrm{e}}$ série, Tome IV, 1873, p. 613-627.

Bekendam R.F., Oldenziel C.E., Paar W.A. - Subsidence potential of the Hengelo brine field (part I). Physico-chemical deterioration and mechanical failure of salt-cavern roof layers. Proceedings of the SMRI fall meeting, San Antonio, 2000, p. 103-117.

Bérest P., Brouard B., Feuga B. - Abandon des mines de sel : faut-il ennoyer? Revue française de géotechnique, $\mathrm{n}^{\circ} 106$ 107,2004, p. 53-71.

Bérest P., Blum P.A., Charpentier J.-P., Gharbi H., Valès F. - Very slow creep tests on rock samples. International Journal Rock Mechanics Mining Sciences, $n^{\circ} 42,2005$, p. 569-576.

Boidin E. - Interactions roches/saumures en contexte d'abandon d'exploitations souterraines de sel. Thèse de doctorat de l'Institut national polytechnique de Lorraine, février 2007

Braconnier M.A. - 1er rapport de l'ingé nieur des mines sur l'effondrement de la mine de sel gemme de VarangévilleSaint-Nicolas, 2 novembre $1873 a$.

Braconnier M.A. - $2^{e}$ rapport de l'ingénieur des mines sur l'effondrement de la mine de sel gemme de Varangéville-SaintNicolas, 3 novembre $1873 \mathrm{~b}$

Braconnier M.A. - $3^{\text {e }}$ rapport de l'ingénieur des mines sur l'effondrement de la mine de sel gemme de Varangéville-SaintNicolas, 4 novembre $1873 \mathrm{c}$.

Buffet A. - The Collapse of Compagnie des Salins SG4 and SG5 Drillings. Proceedings of the SMRI fall meeting, Roma, 1998 , p. $79-105$.
Chan K.S., Munson D.E., Fossum A.F. Bodner S.R. - A constitutive model for representing coupled creep, fracture and healing in rock salt. Proceedings of the 4th conference on the mechanical behavior of salt. Clausthal-Zellerfeld, Germany : Trans Tech Publishers, 1996, p. 221-247.

De Vries K.L., Mellegard K.D., Callahan G.D - Cavern Design Using a Salt Damage Criterion : Proof-of-Concept Research Final Report. Proceedings of the SMR spring meeting, Houston, 2003, p. 1-18.

Feuga B. - Old salt mine at Dieuze (France) revisited 150 years after being abando ned. Proceedings of the SMRI fall meeting, Chester, 2002, p. 114-128.

Feuga B., Bérest P., Karimi-Jafari M. Brouard B. - Mechanical Behaviour of a Marly Floor in two mines where brine or water was abundant. Proceedings of the SMRI fall meeting. Porto, 2008, p. 19-40.

Feuga B. - Les effondrements dus à l'exploitation du sel. Geosciences, $n^{\circ} 9$, avril 2009.

Jeanneau V. - The sinkhole of the cavity LR 50/51 in La Rape Area, a case history. RHODIA Company. Proceedings of the SMRI fall meeting, Nancy, 2005, p. 9-24.

Klein E., Contrucci I., Daupley X., Hernandez O., Bigarré P., Nadim C, Cauvin L., Pirson M. - Experimental monitoring of a solution-mining Cavern in Salt : Identifying and Analyzing Early-Warning Signals Prior to Collapse. Proceedings of the SMRI fall meeting, Austin, 2008, p. 135-146.

Marchal C. - Le gîte salifère keupérien de Lorraine-Champagne et les formations associées. Étude géométrique. Implications génétiques. Mémoire $n^{\circ} 44,2$ volu mes, 1983. Sciences de la Terre, Nancy.
Minkley W., Menzel W. - Local Instability and System Instability of Room and Pillar Fields in Potash Mining. Proceedings of the 3rd conference on the mechanical behavior of salt. Clausthal-Zellerfeld, Germany: Trans Tech Publishers, 1996, p. 497-510.

Minkley W., Mühlbauer J., Storch G. Dynamic processes in salt rocks : a general approach for softening processes within the rock matrix and along the bedding planes. Proceedings of the 6th conference on the mechanical behavior of salt Taylor \& Francis, London UK, 2007, p. 295-304.

Robelin C, Bonijoly D. - Les discontinuités sédimentaires de la série salifère triasique de Varangéville (Meurthe-et Moselle). Rapport BRGM-SGN R 31 834, octobre 1990

Rothenburg L., Dusseault M., Mraz D.Z. - A methodology for rock mechanics design of brine fields based on case histories of sinkhole formation in Windsor-Detroit area. Proceedings of the 5th conference on the mechanical behavior of salt Clausthal-Zellerfeld, Germany : Trans Tech Publishers, 2002, p. 389-392.

Salençon J., Matar M. - Capacité portante des fondations circulaires. Journal de Mécanique Théorique et Appliquée, vol. I, n'2,1982, p. 237-267.

Vouille G. - Mine de Varangéville, Comportement mécanique des marnes du mur en présence de saumure. Rapport R 86/3 de l'École des mines de Paris pour la CSMSE, 1986. 